\title{
Differentiation Unclean and Cleaned Edible Bird's Nest using Multivariate Analysis of Amino Acid Composition Data
}

\author{
Nurul Alia Azmi ${ }^{1}$, Ting Hun Lee ${ }^{1,2 *}$, Chia Hau Lee ${ }^{1}$, Norfadilah Hamdan ${ }^{1}$ and \\ Kian Kai Cheng ${ }^{2}$ \\ ${ }^{1}$ School of Chemical and Energy Engineering, Faculty of Engineering, Universiti Teknologi Malaysia, \\ 81310 UTM, Skudai, Johor, Malaysia \\ ${ }^{2}$ Innovation Centre in Agritechnology for Advanced Bioprocessing (ICA), Universiti Teknologi Malaysia- \\ Pagoh, Jalan Edu Hub UTM 2, Hub Pendidikan Tinggi Pagoh, 84600 Pagoh, Johor, Malaysia
}

\begin{abstract}
Edible Bird's Nest (EBN) has been used as a health modulator for many centuries. Nutrient degradation in EBN always happen during cleaning process due to many factors such as temperature and long soaking time in water. The present study attempts to find the difference between unclean and cleaned EBN in their amino acid composition. A total of $65 \mathrm{EBN}$ samples were collected directly from swiftlet premises in 13 states of Malaysia to ensure the coverage of geographical location differences. A standardized cleaning method had been adapted from the industry to clean the collected EBN sample in the lab. Then it was analysed for amino acids composition. After that OPLS-DA multivariate model was used to discriminate the unclean and cleaned EBN on 18 types of amino acids composition. The model was robust with classification and predictive ability of $76.1 \%$ and $64.5 \%$, respectively. The model was further validated with sample blind test and $100 \%$ of the sample was accurately fall into their respective cluster, unclean and cleaned EBN. The findings suggest that three major amino acids with the highest VIP value were Aspartic acid,

ARTICLE INFO

Article history:

Received: 09 September 2020

Accepted: 05 November 2020

Published: 22 January 2021 Methionine and Glutamic acid and proposed as the marker for discriminating the unclean and cleaned EBN.
\end{abstract}

DOI: https://doi.org/10.47836/pjst.29.1.36

$\overline{\text { E-mail addresses: }}$

nurulalia95@gmail.com (Nurul Alia Azmi)

leetinghun@utm.my (Ting Hun Lee)

leechiahau0506@gmail.com (Chia Hau Lee)

fadilahamdan87@gmail.com (Norfadilah Hamdan)

chengkiankai@utm.my (Kian Kai Cheng)

* Corresponding author
Keywords: Cleaning process, edible bird's nest (EBN), orthogonal partial least square discriminant analysis (OPLS-DA) 


\section{INTRODUCTION}

Edible Bird's Nest (EBN) is produced by the regurgitated secretion of swiftlets, Aerodramus fuchiphagus (Shim et al., 2016). It is a delicacy and food tonic that is popular, especially among the Chinese community all over the world. It has been used as a Traditional Chinese Medicine (TCM) since the Tang Dynasty (618-907) A.D (Lim, 2007; Marcone, 2005). According to the Compendium of Materia Medica "Ben Cao Gang Mu" written by $\mathrm{Li}$ Shizhen, EBN is an energy tonic that reinforces energy, nourishes lung, helps to maintain a youthful and radiant complexion ( $\mathrm{Li} \& \mathrm{Wu}, 2010$ ). Yida et al. (2015) reported that Chinese also used EBN for general well-being purpose such as, fasten the recovery of postpartum for women, enhance renal function, boost the immune system, regulate blood circulation, increase energy and also increase metabolic rate.

EBN is a very complicated industry that involving too many uncertainties, parties, location, or even bird's species are not certainly clear. Apart from the variance that is not able to control by humans such as swiftlets habitat, location and environmental factor, some areas should be closely monitored, and stringent guideline should be recommended to industries. It is the 'gray area' that many malpractices including adulteration and negligent handling of this precious health modulating animal bioproduct especially during the cleaning process. Previously the composition of EBN nutrients was based on different colors, production sites and geographical origins (Halimi et al., 2014; Lukman \& Wibawan, 2018; Norhayati et al., 2010; Saengkrajang et al., 2013; Seow et al., 2016a; Seow et al., 2016b). However, none of these literatures reported the difference content in EBN on unclean and cleaned. The focus should be aimed at the content to provide a clearer understanding on its content. Further investigation on unclean and cleaned EBN should also be researched to see the nutrient change and provide as a guideline to the industries on cleaning or processing to enhance EBN content.

During the early 70's, EBN cleaning industries was a highly kept secrets, and no information was able to obtain from any source including literature. When the house variety of EBN emerged and became popular in the 80's, the EBN cleaning process has become an industry need. Due to lack of research and development, the industry through trial and error searched for an effective cleaning method without considering much on EBN nutrient changes. During that time or earlier, the well-known bleaching technique used a bleaching agent to "clean" the EBN. By using this bleaching technique, the fine feather was bleached to off white and resemble EBN colour. It is efficient, fast, and economy despite not knowing the biochemical properties change and bleaching chemical residual, toxicity and other problem lying beneath. This technique, however, is bad for the consumer. Eventually the cleaning method became unacceptable. Other problem includes excessive soaking in water, steaming and even boiling during the cleaning process might affect some nutrient content change in EBN. It has been reported that EBN is sensitive towards heat, and the composition of its active compounds could be changed (Shim et al., 2016). 
The cleaning process of EBN may trigger the breakdown and denaturation of the polymer such as protein and carbohydrate into amino acids and saccharide, respectively (Chua et al., 2015). It causes the inaccuracy in quantify of the polymer. Based on the literature study, protein is the main component that plays an important role in regulating some metabolism or pathway which contribute to the therapeutic effects (Abidin et al., 2011; Chua et al., 2015; Chua et al., 2013; Chua \& Zukefli, 2016; Wong, 2013). Amino acids are the building block of protein, and these compounds can be a promising indicator for the classification of EBN (Chua et al., 2015; Quek et al., 2018). Results from various researchers and our study have shown that there are 18 types of amino acid detected from EBN and it is well established (Hun et al., 2015; Marcone, 2005; Su et al., 1998). The quantification and qualification of the amino acids are more accurate compared to protein. It is because protein is not stable and easily degraded at high temperature (Chua et al., 2015; Chua et al., 2013). However, at higher temperature (too high), the amino acids itself will denature thus indicating the EBN is over-process and do not have any protein-related nutrient in EBN (Erik et al., 2015). Despite all these discoveries, little is known on the content changes of unclean and cleaned EBN, and to date, the scientific evidence-based studies on this topic have yet been reported. The data on the amino acid composition of unclean and cleaned EBN may give some insights for the better understanding of EBN cleaning process. It is also extremely important to preserve the valuable content of this all rounded therapeutic compound.

This study aimed to investigate the changes of amino acids composition before and after the cleaning process and further identify the amino acids markers for unclean and cleaned EBN. This present study also revealed the distribution of important amino acids. A novel classification method using Orthogonal Partial Latent Square Discriminant Analysis (OPLS-DA) to categorise the unclean and cleaned EBN samples based on its amino acid composition. It is the first study to be reported on an attempt to use the amino acids composition in EBN to differentiate the unclean and cleaned EBN. This finding can be a reference for EBN industries in order to check on their incoming and outgoing product quality. Also, the authority could use these amino acids composition to be a standard to inspect on the malpractice of EBN processors.

\section{MATERIALS AND METHODS}

\section{Sample Preparation}

A total of 65 EBN samples were collected on 1st January to 31st December 2016 from different states in Malaysia ( 5 for each state) to ensure the coverage of geographical location differences. The source for EBN could be an important factor in confirming the variant in EBN constituent. The sample collected from the swiftlet premises were labelled with Radio Frequency Identification marked by Department of Veterinary Services Malaysia. 
However, the identification number is not disclosed here to protect the premises owner's privacy. All samples collected were genuine and directly from the licensed swiftlets premises at a different location that has been used as the reference standard. There were two similar sample set of ENB, consisted of 65 sample each. The first set of EBN undergone the cleaning process with the method according to Good Manufacturing Practice (GMP) of industrial cleaning method [Raw-Unclean and Raw-Clean Edible Bird's Nest (MS 2333:2010)], labelled as cleaned EBN sample. Other set of EBN sample did not go through any cleaning process, labelled as unclean EBN sample. The sample that had undergone cleaning process was ensured to be same as unclean EBN for analysis. This ensured that unclean and cleaned EBN were from the same source and minimized the location variant.

\section{Cleaning Method}

Good Manufacturing Practice (GMP) of industrial cleaning method [Raw-Unclean and Raw-Clean Edible Bird's Nest (MS 2333:2010)] was adapted and standardized for all samples. Briefly, the collected EBN was moisturized with reverse osmosis water, and a pair of forceps was used to separate the feathers, eggshells, dirt and other impurities by human labour. After the cleaning process, the EBN was dried in the oven (Memmert, Schwabach, Germany) at $45^{\circ} \mathrm{C}$ for 2 hours. The total sample for this study was 130 samples, consisting of 65 unclean and cleaned samples each.

\section{Amino Acids Analysis}

All amino acids detection was carried out using acid hydrolysis method except Tryptophan. (Oda et al., 1998; Su et al., 1998). $0.3 \mathrm{~g}$ of ground EBN samples were weighed and transferred into hydrolyzing bottles. $15 \mathrm{ml}$ of $6 \mathrm{~N} \mathrm{HCl}$ was added and sealed tightly. Then, the samples were hydrolyzed in a convection oven (Memmert, Schwabach, Germany) for $24 \mathrm{~h}$ at $40^{\circ} \mathrm{C}$.

While Tryptophan was determined by using alkaline hydrolysis because it is not stable and may destroy in acid condition (Reverter et al., 1997). The ground EBN (0.1 g) was placed in an evacuated tube and added with $15 \mathrm{~mL}$ of $4.3 \mathrm{~N}$ lithium hydroxide ( $\mathrm{LiOH}$. $\mathrm{H} 2 \mathrm{O}$ ). The mixture was flushed with $\mathrm{N} 2$ gas and heated in an oven (Memmert, Schwabach, Germany) at $120^{\circ} \mathrm{C}$ for 16 hours. The $\mathrm{pH}$ of the sample was adjusted to 4.5 , with a diluted hydrochloric acid solution (0.1M).

Derivation step of amino acids was done with the aid of AccQ.Fluor Reagent kit (Waters Corporation, Massachusetts, USA). The derivatized samples were filtered using syringe filter $(0.45 \mu \mathrm{m}$ pore size hydrophilic PVDF) before being injected into the Waters e2695 High-Performance Liquid Chromatography instrument (Waters Corporation, 
Massachusetts, USA), fitted with AccQ. Tag $3.9 \times 150 \mathrm{~mm}$ column (Waters Corporation, Milford, Massachusetts, USA). Then, the detection was done using a Waters 2475 fluorescence detector (Waters Corporation, Milford, Massachusetts, USA) at $254 \mathrm{~nm}$. All targeted compounds were identified base on the retention time of the corresponding standard. Then, they were quantified using the internal standard method adapted from Khaleduzzaman et al. (2008) except Tryptophan. The internal standard used was $\alpha$ Amino Butyric Acid (AABA). The quantity of Tryptophan in sample was compared to a calibration curve of Tryptophan standard.

\section{Statistical Analysis}

SPSS v20 for Windows (SPSS Inc., Chicago United States) was used to analyzed experimental data and subjected to independent sample T-test and Pearson Correlation analysis.

\section{Orthogonal Partial Latent Square Discriminate Analysis}

The chemometric analysis was done using SIMCA software (version 14.1.0, Umetric, Sweden) while Orthogonal Partial Latent Square Discrimination Analysis (OPLS-DA) performed the classification. OPLS-DA is the supervised statistical analysis method that predicts the variable $\mathrm{Y}$ (unclean and cleaned EBN) by explanatory quantitative variables $\mathrm{X}$ (18 types of amino acids). It can separate the systematic variation in $\mathrm{X}$ variable into twopart, one part is $\mathrm{X}$ variable correlated to $\mathrm{Y}$ variable; and second part is $\mathrm{X}$ variable orthogonal to $\mathrm{Y}$ variable, which does not contribute to discrimination of unclean and cleaned EBN (Blasco et al., 2015). The robustness of the model was interpreted after seven-fold internal cross-validation by cumulative goodness of fit $\left(\mathrm{R}^{2} \mathrm{Y}\right)$, goodness of prediction $\left(\mathrm{Q}^{2}\right)$ and Cross-Validation Analysis of Coefficient Variance (CV-ANOVA) (Beauclercq et al., 2019).

OPLS-DA model is statistically reliable if CV-ANOVA, $\mathrm{p}<0.05$. The model can be interpreted as robust if the $\mathrm{Q}^{2}$ (cum) $>0.4$ and $\mathrm{R}^{2} \mathrm{Y}$ (cum) $>0.5$ (Blasco et al., 2015). Each $\mathrm{X}$ variable that contributes to the OPLS-DA model was summarized by Variable Importance Projection (VIP) value. High VIP value indicates, high contribution of the $\mathrm{X}$ variable to discriminate the unclean and cleaned EBN on the OPLS-DA model (Beauclercq et al., 2019). High VIP value combining with small Jackknife confident interval can be potential marker to discriminate the unclean and cleaned EBN sample (Beauclercq et al., 2019). The basic method of Jackknife is by removing one sample in the dataset and replacing it with the blind sample in the population to recompute the mean and obtain the confident interval. The small Jackknife confident interval value indicates that the dataset is approximately correct and has no bias (Blasco et al., 2015). 


\section{RESULT AND DISCUSSION}

\section{Amino Acids Composition}

Figure 1 shows a typical chromatogram of EBN amino acids and ammonia obtained from the acid hydrolysate extract. There were 17 types of amino acids found in the analyzed EBN samples. However, ammonia was not accounted in this study as it is not an amino acid. Besides Figure 2 shows, the chromatogram of EBN sample from alkaline hydrolysate extract. Based on the result, all analyzed EBN samples contained Tryptophan.

Serine was the highest amino acid in unclean EBN with $3.72 \pm 0.36 \mathrm{w} / \mathrm{w} \%$ and followed by Aspartic acid with $3.51 \pm 0.41 \mathrm{w} / \mathrm{w} \%$ (Figure 3). Meanwhile, for cleaned EBN, Phenylalanine was the highest amino acid with $4.21 \pm 0.32 \mathrm{w} / \mathrm{w} \%$ and next most abundant amino acid was Serine with $4.06 \pm 0.42 \mathrm{w} / \mathrm{w} \%$ (Figure 3 ). These results agree with Ali et al. (2019), Chua et al. (2015) and Marcone (2005). Their analyses showed that the most abundant amino acids of EBN were Serine, Aspartic acid, and Phenylalanine.

This study found that Tryptophan, Methionine and Alanine were the lowest amino acids in both unclean and cleaned EBN (Figure 3). These results were in line with the previous finding from Ali et al. (2019) and Chua et al. (2015), where these three amino acids were among the lowest amino acids in EBN. However, this is contradicting to the previous study by Saengkrajang et al. (2013) which found that Methionine was the highest amino acid in EBN. One possible reason that could make the difference is due to the origin of the EBN collected and also the cleaning method employed.

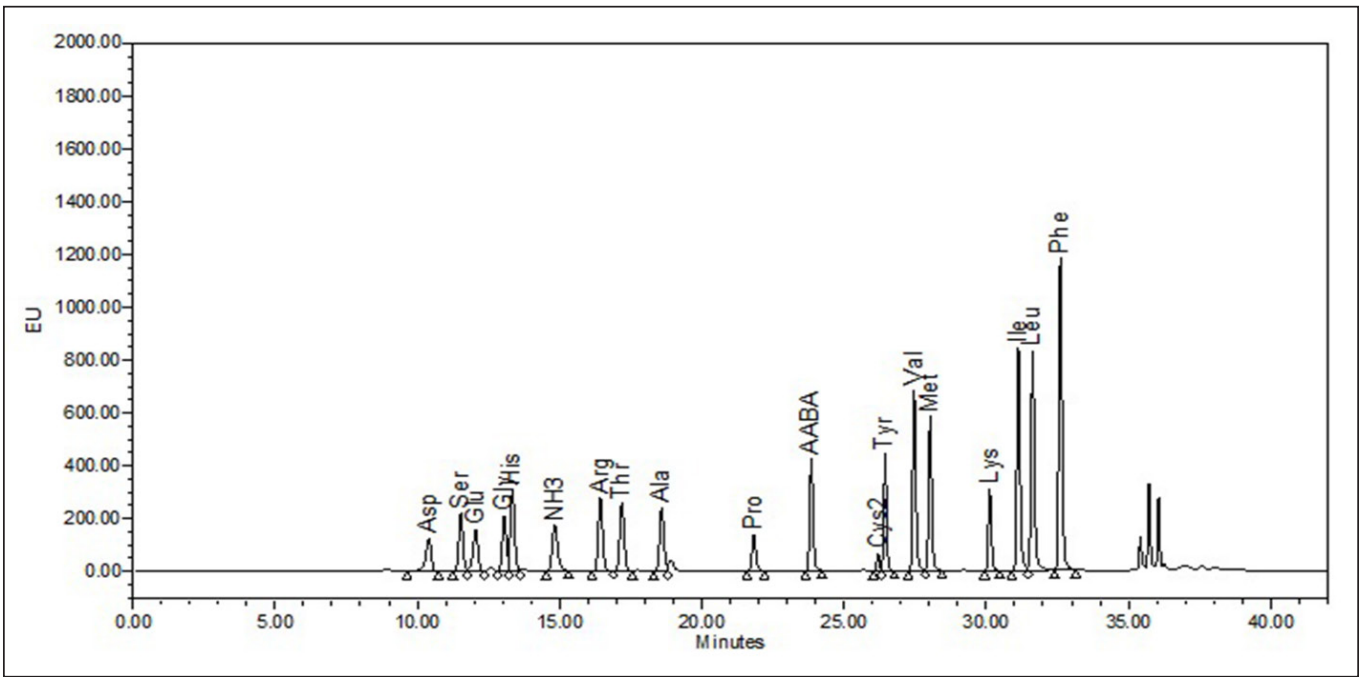

Figure 1. Typical chromatogram of the 17 types of amino acids from acid hydrolysate EBN sample including the internal standard (AABA) and ammonia, adapted from Hun et al. (2020). Abbreviation: Asp, Aspartic acid; Ser, Serine; Glu, Glutamic acid; Gly, Glycine; His; Histidine; NH3, Ammonia; Arg, Arginine; Thr, Threonine; Ala, Alanine; Pro, Proline; AABA, Alpha Amino Butyric Acid, Cys, Cysteine; Tyr, Tyrosine; Val, Valine; Met, Methionine; Lys, Lysine; Ile, Isoleucine; Leu, Leucine; Phe, Phenylalanine 


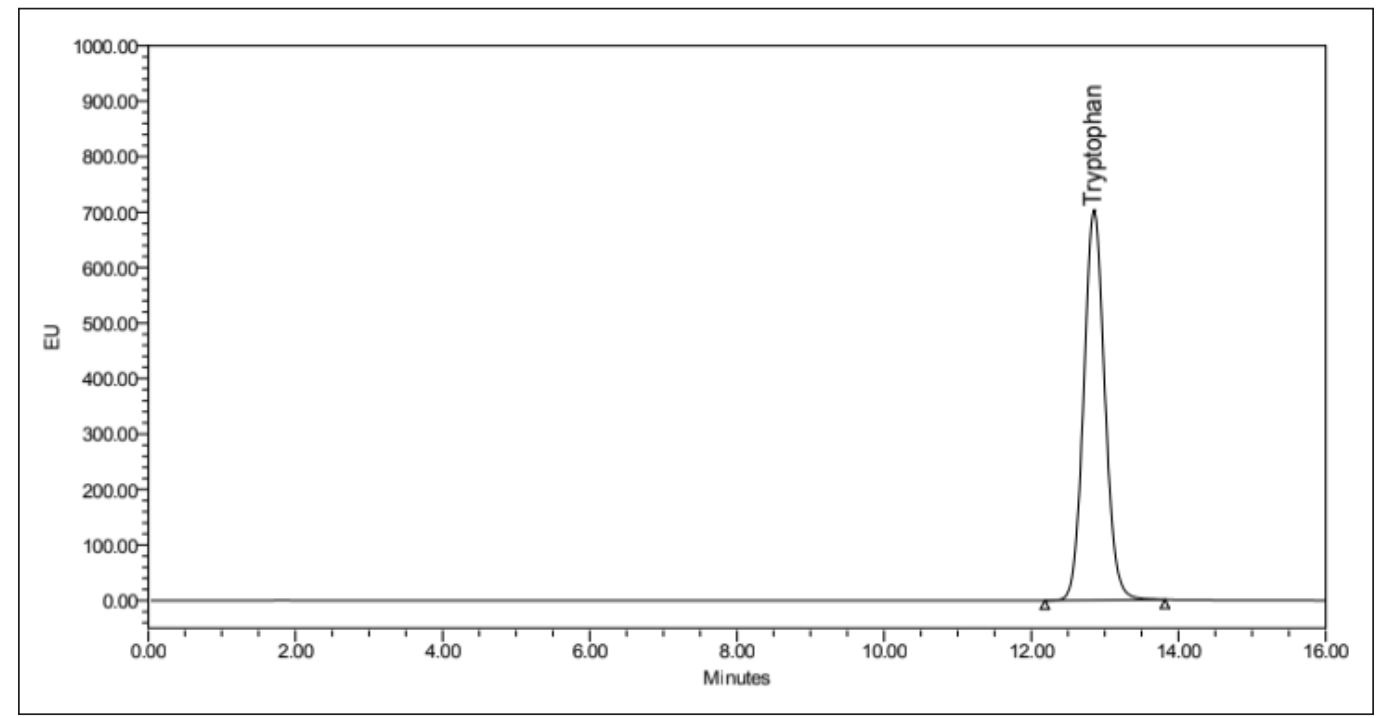

Figure 2. Typical chromatogram of Tryptophan in EBN sample from alkaline hydrolysate extract



Figure 3. Amino acids composition of unclean and cleaned EBN. The data shown the mean of 65 independent samples \pm Standard Deviation (SD). Asterisk (*) represent significant difference between unclean and cleaned $\operatorname{EBN}(\mathrm{p}<0.05)$

\section{Effect of EBN Cleaning Process in Amino Acids Content}

The results obtained revealed that the total amount of amino acid content in cleaned EBN was significantly higher $(\mathrm{p}<0.05)$ compared to unclean EBN with $44.8 \pm 1.05 \mathrm{w} / \mathrm{w} \%$ $(n=65)$ and $40.4 \pm 1.32 \mathrm{w} / \mathrm{w} \%(\mathrm{n}=65)$ respectively. Figure 3 shows 18 amino acids content in unclean and cleaned EBN samples. From the result obtained, this study shows that the cleaning process affected the amino acids content in EBN. This study revealed most individual amino acid content increased after cleaning process except Lysine, Aspartic 
acid, Glutamic acid, Alanine and including Tryptophan, although it showed very little difference in content. The increment of amino acids content could be due to the key factor of drying temperature $\left(40^{\circ} \mathrm{C}\right)$ during the cleaning process that breakdown the protein into amino acids. The heat could cause additional energy that changed the protein structure, characteristic and molecule arrangement (Mauer, 2003). This present study is in agreement with Aluko (2018) that found the optimum temperature to breakdown the protein into amino acids was between $37^{\circ} \mathrm{C}$ to $50^{\circ} \mathrm{C}$.

There are some amino acids content decreased significantly after the cleaning process including, Lysine, Aspartic acid, and Glutamic acid. According to Erik et al. (2015), Aspartic acid and Glutamic acid are categorized under charged and acidic amino acid and, these types of amino acids contain high propensity and energetically favorable contact with water Furthermore, data from Del et al. (2009)., found that, these amino acids contained lateral chain that allowed the formation of zwitterionic function to solubilize the amino acid in water. The zwitterionic formed when an amino acid has the amino group (negative charge) and carboxylic group (positive charge) in ion form. During the cleaning process, a large amount of water was in contact with the EBN for 5-6 hours. This could be the major reason that promotes Aspartic acid and Glutamic acid to dissolve into water. Tripathy et al. (2018) revealed that Glutamic acid had good solubility in water compared to other amino acids, and it could be leached when contact with water during the cleaning process of EBN. Lysine in cleaned EBN was also significantly lower compared to unclean EBN. This might due, this amino acid is sensitive to multi stimuli such as temperature and $\mathrm{pH}$ (Tripathy et al., 2018). A study done by Tulbek et al. (2017) found that, the Lysine content in cooked pea bean was significantly lower compared to uncook pea bean. It could be the reason that, why Lysine content decreased after the cleaning process.

The EBN cleaning process has made the content of the amino acids changed significantly (Figure 3). This showed that it is important to monitor the key compound of EBN before and after the cleaning process. There were 15 amino acids that changed significantly, and only 3 are not. Further research on the molecular level could be explored to find the theory behind this.

\section{Discrimination of Unclean and Cleaned EBN}

The OPLS-DA model performance was based on 18 types of amino acids content from unclean and cleaned EBN. Figure 4 shows $85 \%$ of the randomly selected original data (55 samples each from both classes, 110 samples) as a predictive model and remaining $15 \%$ of the sample was used for testing set to project into predictive model to validate the accuracy (Seow et al., 2016a). The predictive variation given by $\left(\mathrm{R}^{2} \mathrm{X}_{\text {cum }}\right)$ between $\mathrm{X}$ (amino acids) and Y (EBN sample), the best model used five components, and interprets $95 \%$ of the total variation in X. OPLS-DA developed a three-component model based on the data set. In 
addition, robustness of the model explained by $76.1 \%$ of the differences between the two groups $\left(\mathrm{R}^{2} \mathrm{Y}_{\text {cum }}\right)$, clearly separating the unclean and cleaned EBN samples. The model has high predictive ability with $64.5 \%\left(\mathrm{Q}^{2}\right.$ cum $)$ and it was validated by cross-validated variance analysis (CV-ANOVA) with a P-value of 6.07/1020. Seven-fold cross-validation used as internal validating method to validate the prediction ability of the model (Beauclercq et al., 2019). Figure 4 shows, the model plot of score scatters indicate that sample classification was highly sensitive to classified unclean and cleaned EBN. Repetitive dots of different groups mapped on OPLS-DA model score plot showed a strong separation suggesting that the unclean and cleaned EBN could be differentiated based on their respective amino acids observed.

Several studies have reported on the application of OPLS-DA method to classify the distinctive category of the sample (Cavanna et al., 2019; Phua et al., 2014; Song et al., 2013). This shows that the OPLS-DA is robust and reliable to separate the sample according to the specific category. Phua et al. (2014) reported an important discovery where they classified the colorectal cancer cell and the healthy cell based on the metabolite compounds by using OPLS-DA. Cavanna et al. (2019) successfully classified the chicken egg based on its freshness. The most recent application of OPLS-DA on EBN sample was reported by Seow et al. (2016a) where they successfully discriminated the cave and house EBN with predictive power $\left(\mathrm{Q}^{2}\right.$ cum $)$ of $76.1 \%$. The developed OPLS-DA model from our study and Seow et al. (2016a) can be used to classify unknown EBN sample in future.

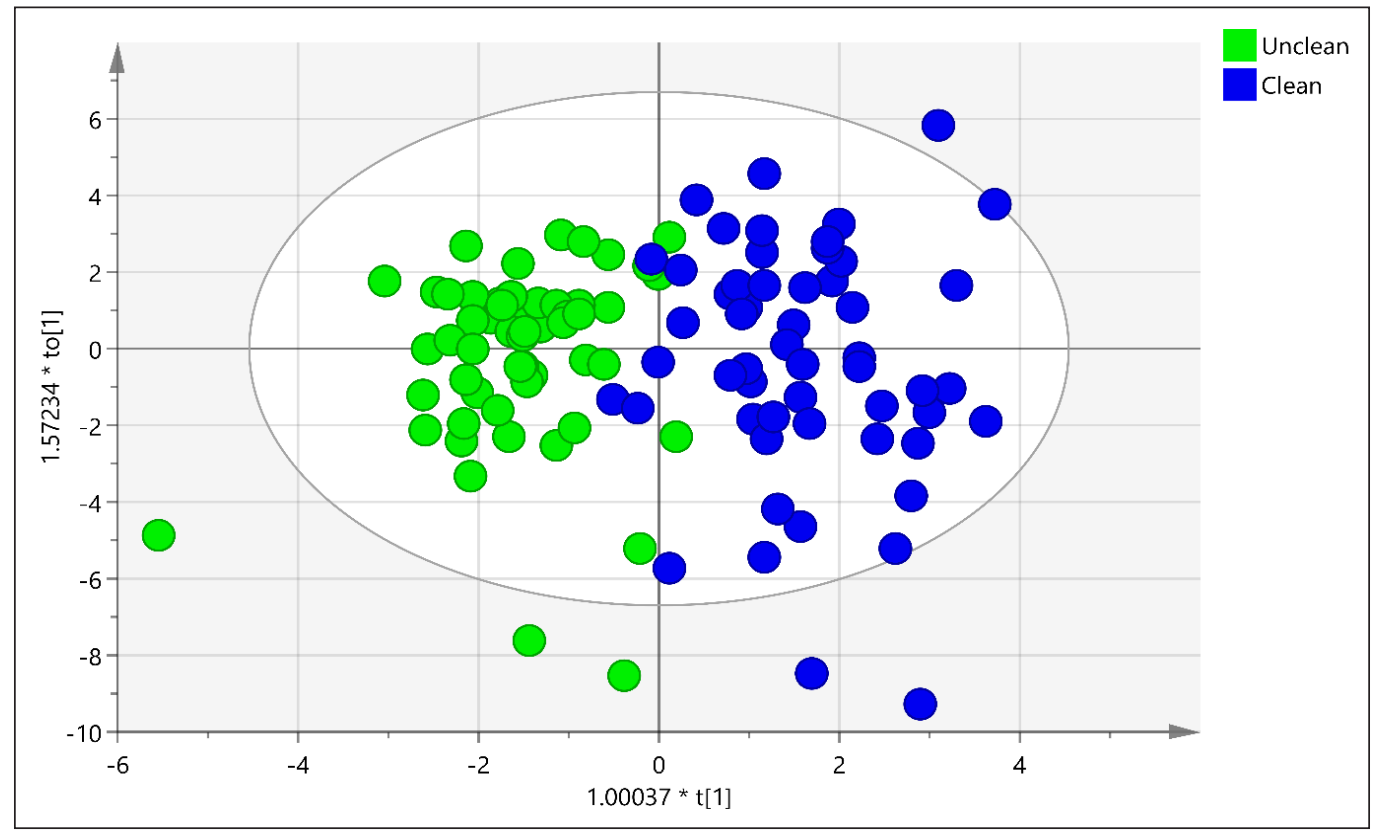

Figure 4. OPLS-DA model score plot for $85 \%$ training set $(\mathrm{n}=110)$ of 18 variables (amino acids) for unclean and cleaned EBN sample. A score plot for unclean EBN ( $\mathrm{n}=55$, green dot) and cleaned EBN ( $\mathrm{n}=55$, blue dot) 


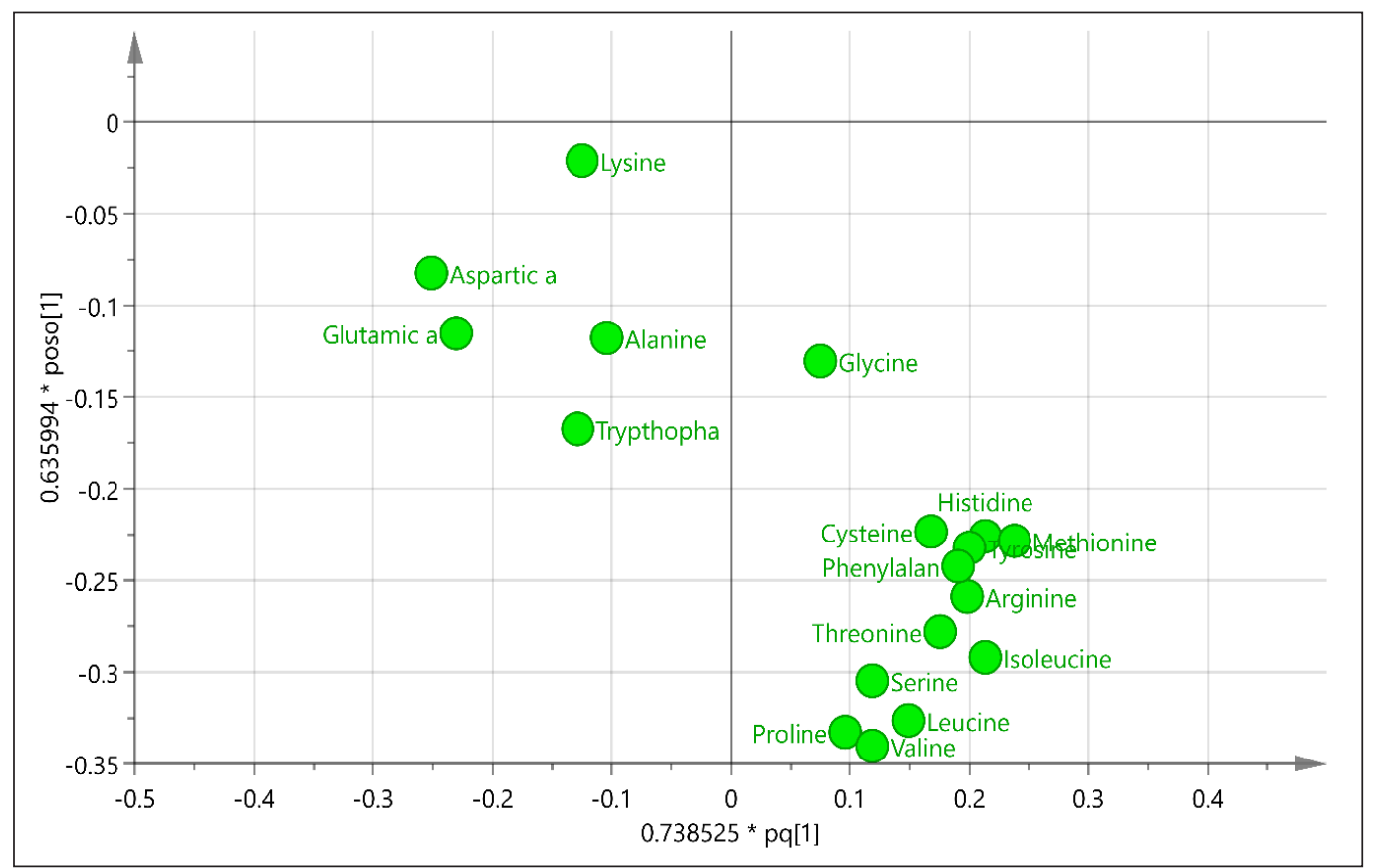

Figure 5. Loading plot for OPLS-DA overview based on the OPLS-DA model including 18 type of amino acids

Figure 5 shows the loading plot of the model, where variable was expressed differently between unclean and cleaned EBN samples to confirm the score plot result. Five amino acids Lysine, Aspartic acid, Glutamic acid, Alanine and Tryptophan are on the same side of the unclean class in the score plot of the model (Figure 4). It was shown that these five amino acids were dominant in unclean EBN. Data have shown that these five amino acids were reduced after the cleaning process (Figure 3).

\section{Evaluation of Predictive Model Robustness and Maker Validation for Discrimination of Unclean and Cleaned EBN}

The predictive model robustness was assessed by using external validation and the remaining $15 \%$ of the 130 EBN sample as a testing set. They have been treated as 'unknown' sample to validate the sample classification in exact cluster and not overfitting to the predictive model. The model classified all 20 samples correctly (Figure 6) and the 10 each unclean and cleaned EBN samples were grouped into their respective clusters exactly. Blind test sample classification accuracy was 100\%, and the 'unknown' EBN samples fall into their exact cluster.

Figure 7 shows the value of Variable Projection Influence (VIP) suggested weightage of each variable within the model to effectively differentiate between the two classes on the basis of their differences. Variables that are weak predictive reliability, whether with low VIP value magnitude or with their Jackknife confidence interval crossing zero, are low 


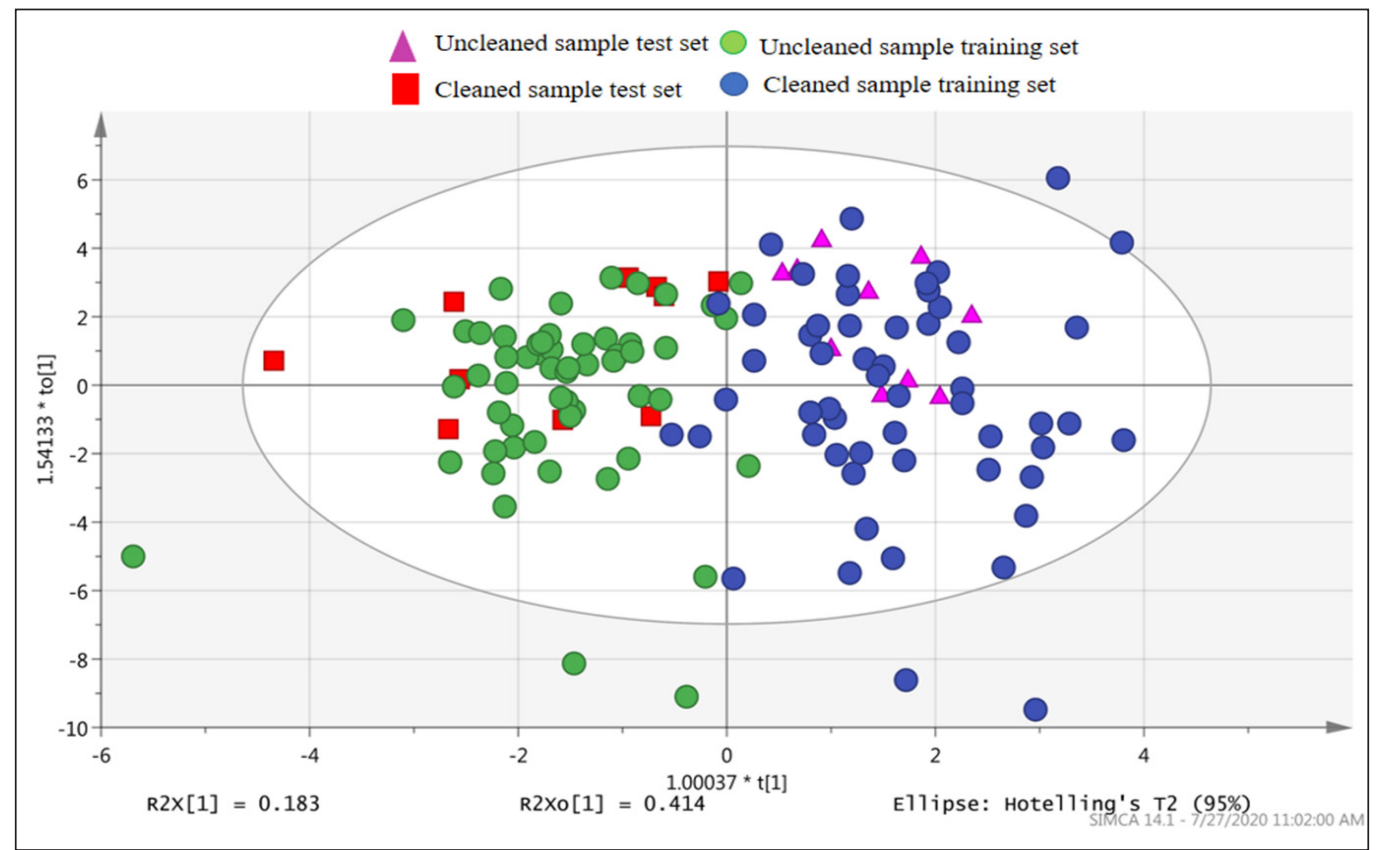

Figure 6. Predicted score plot for $15 \%$ testing set and $85 \%$ of training set of EBN samples. A total of 20 unclean and cleaned samples test set fell into their accurate classes

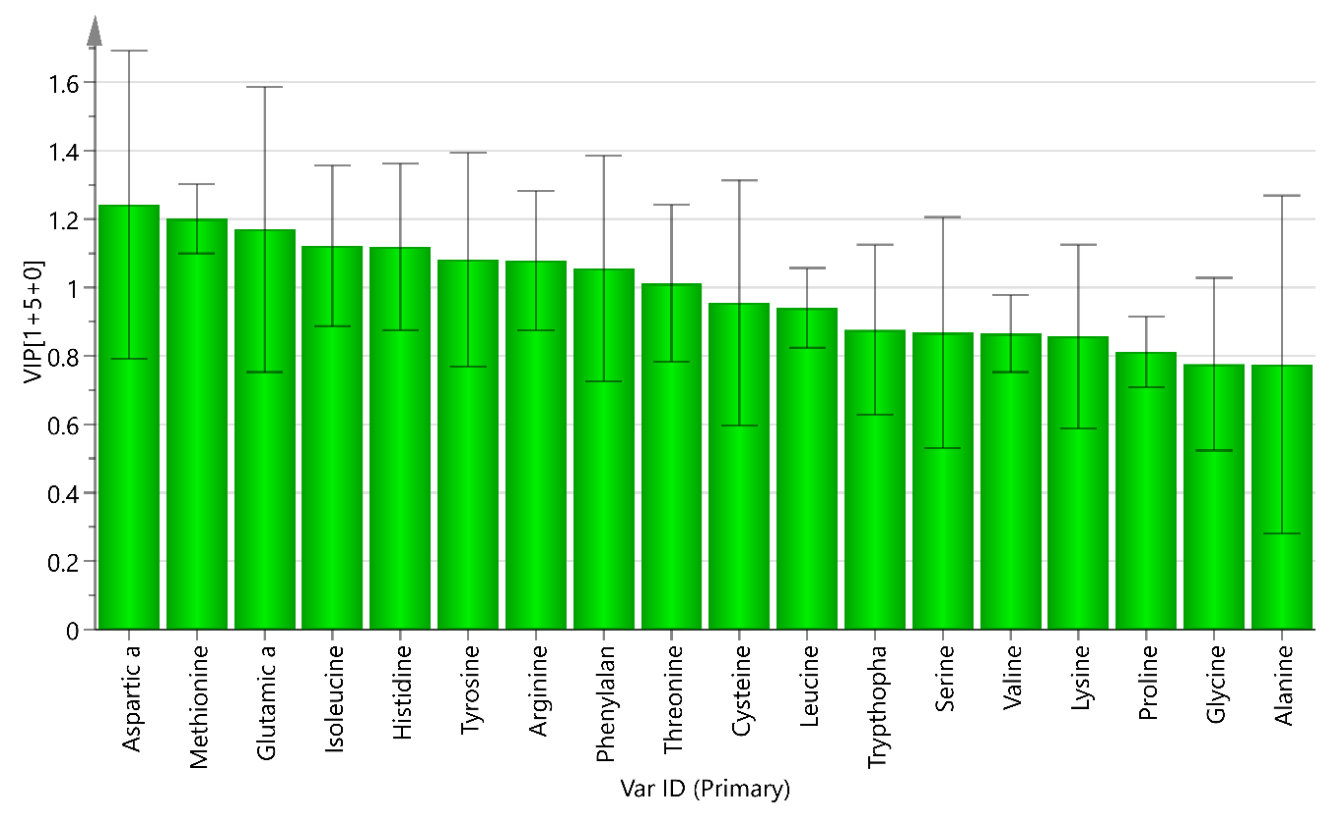

Figure 7. Variable on Projection (VIP) plot of OPLS-DA with Jackknife confident interval. The variables were sort from the highest to lowest VIP value. The highest VIP value indicates the most important variable for discriminating unclean and cleaned EBN sample into the OPLS-DA model score plot 
reliability in prediction and not robust (Seow et al., 2016a). VIP was then used to select those compounds possessing the highest discrimination i.e. unclean and cleaned potential in the predictive model (VIP score $>1$ ). The three highest VIP variables namely Aspartic acid, Methionine and Glutamic acid were proposed as primary markers that could be used to differentiate unclean and cleaned EBN (Blasco et al., 2015; Marcone, 2005; Senizza et al., 2019). Based on the predictive model and the blind test results, the primary markers are valid.

\section{CONCLUSION}

The EBN cleaning process has changed the amino acid composition of EBN. It was found that only Glutamic acid, Aspartic acid, and Lysine were significantly decreased after the cleaning process whereases the others have increased. Eighteen types of amino acids were found in both unclean and cleaned EBN samples. EBN can be differentiated between unclean and cleaned based on their amino acid composition. The model developed has successfully distinguished and further classified the EBN samples into unclean and cleaned classes based on amino acids composition. Aspartic acid, Methionine and Glutamic acid can be used as the markers with the highest discriminative weightage to distinguish between the unclean and cleaned EBN samples according to its class as proposed by the model developed in this study.

\section{ACKNOWLEDGMENT}

The authors would like to thank Universiti Teknologi Malaysia (UTM) for providing necessary facilities to complete experimental work. The research was funded by Universiti Teknologi Malaysia (UTM) via research grant (R.J130000.7846.4J273). The authors also would like to thank John Wiley and Sons Publisher for giving us the permission to re-use the Figure 1 image from the manuscript entitled "Characterization of Polar and Non-Polar Compounds of House Edible Bird’s Nest (EBN) from Johor, Malaysia” (Hun et al., 2020).

\section{REFERENCES}

Abidin, F. Z., Hui, C. K., Luan, N. S., Ramli, E. S. M., Hun, L. T., \& Ghafar, N. A. (2011). Effects of edible bird's nest (EBN) on cultured rabbit corneal keratocytes. BMC Complementary and Alternative Medicine, 11(1), 1-10. doi: https://doi.org/10.1186/1472-6882-11-94

Ali, A. A. M., Noor, H. S. M., Chong, P., Babji, A. S., \& Lim, S. (2019). Comparison of amino acids profile and antioxidant activities between edible bird nest and chicken egg. Malaysian Applied Biology, 48(2), 63-66.

Aluko, R. (2018). Food protein-derived peptides: Production, isolation, and purification. In Proteins in Food Processing (2nd Ed., pp. 389-412). Amsterdam, Netherlands: Woodhead Publishing. doi: https://doi. org/10.1016/B978-0-08-100722-8.00016-4 
Beauclercq, S., Lefèvre, A., Montigny, F., Collin, A., Tesseraud, S., Leterrier, C., ... \& Guilloteau, L. A. (2019). A multiplatform metabolomic approach to characterize fecal signatures of negative postnatal events in chicks: A pilot study. Journal of Animal Science and Biotechnology, 10(1), 1-12. doi: https:// doi.org/10.1186/s40104-019-0335-8

Blasco, Błaszczyński, J., Billaut, J. C., Nadal-Desbarats, L., Pradat, P. F., Devos, D., .. \& Corcia, P. (2015). Comparative analysis of targeted metabolomics: Dominance-based rough set approach versus orthogonal partial least square-discriminant analysis. Journal of Biomedical Informatics, 53, 291-299. doi: https:// doi.org/10.1016/j.jbi.2014.12.001

Cavanna, D., Zanardi, S., Dall'Asta, C., \& Suman, M. (2019). Ion mobility spectrometry coupled to gas chromatography: A rapid tool to assess eggs freshness. Food Chemistry, 271, 691-696. doi: https://doi. org/10.1016/j.foodchem.2018.07.204

Chua, Y. G., Chan, S. H., Bloodworth, B. C., Li, S. F. Y., \& Leong, L. P. (2015). Identification of edible bird's nest with amino acid and monosaccharide analysis. Journal of Agricultural and Food Chemistry, 63(1), 279-289. doi: https://doi.org/10.1021/jf503157n

Chua, K. H., Lee, T. H., Nagandran, K., Yahaya, N. H. M., Lee, C. T., Tjih, E. T. T., \& Aziz, R. A. (2013). Edible bird's nest extract as a chondro-protective agent for human chondrocytes isolated from osteoarthritic knee: In vitro study. BMC Complementary and Alternative Medicine, 13(1), 1-9. doi: https://doi. org/10.1186/1472-6882-13-19

Chua, L. S., \& Zukefli, S. N. (2016). A comprehensive review of edible bird nests and swiftlet farming. Journal of Integrative Medicine, 14(6), 415-428. doi: https://doi.org/10.1016/S2095-4964(16)60282-0

Del, C. C. P., Garde-Cerdán, T., Sánchez, A. M., Maggi, L., Carmona, M., \& Alonso, G. L. (2009). Determination of free amino acids and ammonium ion in saffron (Crocus sativus L.) from different geographical origins. Food Chemistry, 114(4), 1542-1548. doi: https://doi.org/10.1016/j.foodchem.2008.11.034

Erik, V. D., Hoogeveen, A., \& Abeln, S. (2015). The hydrophobic temperature dependence of amino acids directly calculated from protein structures. PLoS Computational Biology, 11(5), 1-17. doi: https://doi. org/10.1371/journal.pcbi.1004277

Halimi, N. M., Kasim, Z. M., \& Babji, A. S. (2014). Nutritional composition and solubility of edible bird nest (Aerodramus fuchiphagus). In AIP Conference Proceedings (Vol. 1614, No. 1, pp. 476-481). New York, USA: AIP Publishing LLC.

Hun, L. T., Lee, C. H., Azmi, N. A., Kavita, S., Wong, S., Znati, M., \& Jannet, H. B. (2020). Characterization of polar and non-polar compounds of house edible bird's nest (EBN) from Johor, Malaysia. Chemistry and Biodiversity, 17(1), 1-10. doi: https://doi.org/10.1002/cbdv.201900419

Hun, L. T., Wani, W. A., Tjih, E. T. T., Adnan, N. A., Ling, Y. L., \& Aziz, R. A. (2015). Investigations into the physicochemical, biochemical and antibacterial properties of edible bird's nest. Journal of Chemical and Pharmaceutical Research, 7(7), 228-247.

Khaleduzzaman, A., Khandaker, Z., Khan, M., Banu, L., \& Khan, M. (2008). Evaluation of a high performance liquid chromolography (Hplc) mehtod for amino acid analysis in feed with precolumn derivatization and fluorescence detection. Bangladesh Journal of Animal Science, 37(2), 66-73. doi: https://doi.org/10.3329/ bjas.v37i2.9883 
Li, Y., \& Wu, Y. L. (2010). A golden phoenix arising from the herbal nest-A review and reflection on the study of antimalarial drug Qinghaosu. Frontiers of Chemistry in China, 5(4), 357-422. doi: https://doi. org/10.1007/s11458-010-0214-5

Lim, C. (2007). Make millions from swiftlet farming: A definitive guide. Kuala Lumpur, Malaysia: True Wealth.

Lukman, W., \& Wibawan, I. W. T. (2018). Protein profile of edible bird's nest origin Kalimantan and Java Islands Indonesia. Journal of Agriculture and Veterinary Sciences, 11(5), 69-73. doi: 10.9790/2380-1105026973

Marcone, M. F. (2005). Characterization of the edible bird's nest the "Caviar of the East". Food Research International, 38(10), 1125-1134. doi: https://doi.org/10.1016/j.foodres.2005.02.008

Mauer, L. (2003). Heat treatment for food proteins. In Protein (pp. 4868-4872). Amsterdam, Netherland: Woodhead Publishing.

Norhayati, M. K., Azman, O., \& Nazaimoon, W. M. (2010). Preliminary study of the nutritional content of Malaysian edible bird's nest. Malaysian Journal of Nutrition, 16(3), 389-396.

Oda, M., Ohta, S., Suga, T., \& Aoki, T. (1998). Study on food components: The structure of N-linked asialo carbohydrate from the edible bird's nest built by Collocalia fuciphaga. Journal of Agricultural and Food Chemistry, 46(8), 3047-3053. doi: https://doi.org/10.1021/jf980094k

Phua, L. C., Chue, X. P., Koh, P. K., Cheah, P. Y., Ho, H. K., \& Chan, E. C. Y. (2014). Non-invasive fecal metabonomic detection of colorectal cancer. Cancer Biology and Therapy, 15(4), 389-397. doi: https:// doi.org/10.4161/cbt.27625

Quek, M. C., Chin, N. L., Yusof, Y. A., Law, C. L., \& Tan, S. W. (2018). Pattern recognition analysis on nutritional profile and chemical composition of edible bird's nest for its origin and authentication. International Journal of Food Properties, 21(1), 1680-1696. doi: https://doi.org/10.1080/10942912.20 18.1503303

Reverter, M., Lundh, T., \& Lindberg, J. E. (1997). Determination of free amino acids in pig plasma by precolumn derivatization with 6-N-aminoquinolyl-N-hydroxysuccinimidyl carbamate and high-performance liquid chromatography. Journal of Chromatography B: Biomedical Sciences and Applications, 696(1), 1-8. doi: https://doi.org/10.1016/S0378-4347(97)00217-X

Saengkrajang, W., Matan, N., \& Matan, N. (2013). Nutritional composition of the farmed edible bird's nest (Collocalia fuciphaga) in Thailand. Journal of Food Composition and Analysis, 31(1), 41-45. doi: https:// doi.org/10.1016/j.jfca.2013.05.001

Senizza, B., Rocchetti, G., Ghisoni, S., Busconi, M., De Los Mozos Pascual, M., Fernandez, J. A., ... \& Trevisan, M. (2019). Identification of phenolic markers for saffron authenticity and origin: An untargeted metabolomics approach. Food Research International, 126(2019), 1-7. doi: https://doi.org/10.1016/j. foodres.2019.108584

Seow, E. K., Ibrahim, B., Muhammad, S. A., Lee, L. H., \& Cheng, L. H. (2016a). Differentiation between house and cave edible bird's nests by chemometric analysis of amino acid composition data. LebensmittelWissenschaft and Technologie - Food Science and Technology, 65, 428-435. doi: https://doi.org/10.1016/j. lwt.2015.08.047 
Seow, E. K., Ibrahim, B., Muhammad, S. A., Lee, L. H., Lalung, J., \& Cheng, L. H. (2016b). Discrimination between cave and house-farmed edible bird's nest based on major mineral profiles. Pertanika Journal of Tropical Agricultural Science, 39(2), 181-195.

Shim, E. K., Chandra, G. F., Pedireddy, S., \& Lee, S. Y. (2016). Characterization of swiftlet edible bird's nest, a mucin glycoprotein, and its adulterants by Raman Microspectroscopy. Journal of Food Science and Technology, 53(9), 3602-3608. doi: https://doi.org/10.1007/s13197-016-2344-3

Song, H. H., Kim, D. Y., Woo, S., Lee, H. K., \& Oh, S. R. (2013). An approach for simultaneous determination for geographical origins of Korean Panax ginseng by UPLC-QTOF/MS coupled with OPLS-DA models. Journal of Ginseng Research, 37(3), 341-348. doi: 10.5142/jgr.2013.37.341

Su, S. C., Yu, P. C., Liu, C. H., Shiau, H. W., Lee, S. C., \& Chou, S. S. (1998). Application of capillary electrophoresis for identification of the authenticity of bird's nests. Journal of Food and Drug Analysis, 6(1), 455-464.

Tripathy, D. B., Mishra, A., Clark, J., \& Farmer, T. (2018). Synthesis, chemistry, physicochemical properties and industrial applications of amino acid surfactants: A review. Comptes Rendus Chimie, 21(2), 112-130. doi: https://doi.org/10.1016/j.crci.2017.11.005

Tulbek, M., Lam, R., Asavajaru, P., \& Lam, A. (2017). Pea: A sustainable vegetable protein crop. In Sustainable Protein Sources (pp. 145-164). Amsterdam, Netherland: Woodhead Publishing. doi: https:// doi.org/10.1016/B978-0-12-802778-3.00009-3

Wong, R. S. (2013). Edible bird's nest: Food or medicine. Chinese Journal of Integrative Medicine, 19(9), 643-649. doi: https://doi.org/10.1007/s11655-013-1563-y

Yida, Z., Imam, M. U., Ismail, M., Ooi, D. J., Sarega, N., Azmi, N. H., .. \& Yusuf, N. B. (2015). Edible bird's nest prevents high fat diet-induced insulin resistance in rats. Journal of Diabetes Research, 2015, 1-11. doi: https://doi.org/10.1155/2015/760535 
